\title{
ROSE-X: an annotated data set for evaluation of 3D plant organ segmentation methods
}

\author{
Helin Dutagaci ${ }^{1}$, Pejman Rasti ${ }^{1,2,3}$, Gilles Galopin ${ }^{2}$ and David Rousseau ${ }^{1,2^{*}}$
}

\begin{abstract}
Background: The production and availability of annotated data sets are indispensable for training and evaluation of automatic phenotyping methods. The need for complete 3D models of real plants with organ-level labeling is even more pronounced due to the advances in 3D vision-based phenotyping techniques and the difficulty of full annotation of the intricate 3D plant structure.

Results: We introduce the ROSE-X data set of 11 annotated 3D models of real rosebush plants acquired through $X$-ray tomography and presented both in volumetric form and as point clouds. The annotation is performed manually to provide ground truth data in the form of organ labels for the voxels corresponding to the plant shoot. This data set is constructed to serve both as training data for supervised learning methods performing organ-level segmentation and as a benchmark to evaluate their performance. The rosebush models in the data set are of high quality and complex architecture with organs frequently touching each other posing a challenge for the current plant organ segmentation methods. We report leaf/stem segmentation results obtained using four baseline methods. The best performance is achieved by the volumetric approach where local features are trained with a random forest classifier, giving Intersection of Union (loU) values of $97.93 \%$ and $86.23 \%$ for leaf and stem classes, respectively.

Conclusion: We provided an annotated 3D data set of 11 rosebush plants for training and evaluation of organ segmentation methods. We also reported leaf/stem segmentation results of baseline methods, which are open to improvement. The data set, together with the baseline results, has the potential of becoming a significant resource for future studies on automatic plant phenotyping.
\end{abstract}

Keywords: X-ray, Rosebush, Segmentation, Database, Machine learning

\section{Background}

Recent agricultural and genetic technologies require high throughput phenotyping systems which can benefit significantly from the automation of inspection and measurement. Automatic plant phenotyping through 3D data has been a recent research topic in computer vision; however, the scarcity of labeled and complete models of real plants is a roadblock for applying recent machine learning techniques that rely on a vast amount of annotated data. Also, benchmarking data sets are indispensable

\footnotetext{
*Correspondence: david.rousseau@univ-angers.fr

2 INRA, UMR1345 Institut de Recherche en Horticulture et Semences, 42 Georges Morel CS 60057, 49071 Beaucouze, France

Full list of author information is available at the end of the article
}

for proper comparison of current and future phenotyping methods that operate on $3 \mathrm{D}$ data such as volumetric models or point clouds.

The production of annotated data sets has become even more important since the recent bloom of deep learning [1], performance of which was shown to be notably boosted by the availability of large annotated data sets [2]. The success of deep learning methods has triggered the interest in data collection and labeling in specific applications of computer vision such as plant imaging [3]. Most of the freely available annotated plant shoot data sets so far have been in the form of collections of $2 \mathrm{D}$ images acquired in the visible spectrum from top or side view. Among the available 2D data

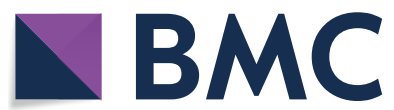

(c) The Author(s) 2020. This article is licensed under a Creative Commons Attribution 4.0 International License, which permits use, sharing, adaptation, distribution and reproduction in any medium or format, as long as you give appropriate credit to the original author(s) and the source, provide a link to the Creative Commons licence, and indicate if changes were made. The images or other third party material in this article are included in the article's Creative Commons licence, unless indicated otherwise in a credit line to the material. If material is not included in the article's Creative Commons licence and your intended use is not permitted by statutory regulation or exceeds the permitted use, you will need to obtain permission directly from the copyright holder. To view a copy of this licence, visit http://creativeco mmons.org/licenses/by/4.0/. The Creative Commons Public Domain Dedication waiver (http://creativecommons.org/publicdomain/ zero/1.0/) applies to the data made available in this article, unless otherwise stated in a credit line to the data. 
sets reported in [3] some are provided with annotated ground truth $[4,5]$, which is very valuable for phenotyping through computer vision and machine learning. In this article, we are interested in providing $3 \mathrm{D}$ annotated models of plants.

Among the most related data sets, some provide multiple images of plants that would allow 3D reconstruction; however, they do not include complete 3D plant models [6-9]. Uchiyama et al. [7] provided a data set containing multiple RGB and depth images of Komatsuna plant together with the manually annotated leaf labels. The data set contains calibration images to be used for estimating 3D geometry from the plant images. Cruz et al. [8] constructed a database named "MSU-PID" containing fluorescence, IR, RGB, and top view depth images of Arabidopsis and bean plants. 3D reconstructions of plants are not available in the database. Bernotas et al. [9] provided an annotated Arabidopsis data set with 3D information acquired using the photometric stereo technique. The data set includes 221 manually annotated Arabidopsis rosettes, which are partially reconstructed using only top-down views of the plants, providing 2.5D information rather than full 3D models. Wen at al. [10] introduced a database of the 3D models of plants and organs from different species, cultivars, and multiple growth periods, however, at present, the majority of the models in the data set correspond to isolated organs, such as models of single leaves or fruits, rather than full plants.

Due to the improvement of the sensitivity of the sensors and the democratization of the technology, X-ray Computer Tomography (CT) is now widely used for plant imaging [11]. While X-ray imaging is the most adopted tool to monitor roots in real soil conditions [12], it is also being employed for the characterization of the aerial parts of plants [13-19]. The use of X-ray imaging has focused on the acquisition of very thin parts enhanced with dye $[13,17,18]$ or the internal $3 \mathrm{D}$ analysis of the aerial part [14-16, 19].

Rosebushes have been studied with computer vision techniques applied on LiDAR and RGB image data [20, $21]$ to produce global characterization of the shoot and from there estimate its ornamental value. In contrast to these optics-based methods, X-ray CT imaging, although more expensive, provides complete and occlusion-free volumetric information of the $3 \mathrm{D}$ geometric structure of the shoot. Such accurate imaging that is able to capture internal structures provides a means to construct full 3D models of real plants. These models can later be used to guide computer vision and pattern recognition techniques that can operate on data acquired with low-cost imaging devices to inspect a large number of plants used in typical phenotyping experiments.
We provide the ROSE-X data set of 11 complete 3D models of real potted rosebush plants with complex architecture acquired through X-ray computed tomography. The rosebushes we captured through X-ray CT imaging have complex architecture and show significantly high amounts of self-occlusion from all viewpoints, i.e., they possess major challenges for optics-based 3D plant reconstruction methods. These models are suitable to be transformed to other data structures, e.g., full or partial point clouds corresponding to the visible surface of the shoot, similar to what would be obtained with optical systems used for 3D reconstruction of plant shoot such as LiDAR or Time-of-Flight (ToF) cameras [22]. This conversion will make it possible to train and evaluate algorithms that operate on point clouds originating from the visible surface. In addition, with the data available for the occluded parts, these models will make it possible to design algorithms that predict complex plant architectural structure from incomplete input.

The 3D voxel space of each rosebush in the data set is fully annotated through labeling each voxel with its corresponding botanical organ class; "organ" referring to the plant units such as leaves, branches, and flowers. Such ground truth data facilitate the detailed description of the architecture and morphology of the plant, and can be used to train automatic phenotyping algorithms aiming to extract both architectural and organ-level traits. Architectural and organ-level trait analysis of 3D data requires an initial stage of classification of points into their respective categories. Current practice is to segment the acquired data of the plant shoot into branches and leaves. In this paper, we focus on leaf-stem segmentation algorithms as one of the phenotyping applications where our data set can serve both as training data and as a benchmark. We chose four representative methods for stem-leaf segmentation: (1) unsupervised classification using local features from point clouds, (2) support vector machine (SVM) classification using local features from point clouds, (3) random forest-based classification of local features from volumetric data, and (4) 3D U-Net applied on volumetric data. The later two were not previously applied to 3D plant organ segmentation problem. We trained and evaluated the methods on the new ROSE$\mathrm{X}$ data set, and provided baseline performance results.

\section{Methods \\ The ROSE- $X$ data set}

We introduce an open repository of complete 3D models of real rosebush plants with ground truth annotations at organ-level. The acquisition was performed through a 3D Siemens X-ray imaging system with a voltage range of $10-450 \mathrm{kV}$, using a tungsten transmission target and a 280-mA current. For this study, the system was operated 
with an $80-\mathrm{kV}$ voltage. The number of projections was 900 , and each radiograph was an average of three exposures of $333 \mathrm{~ms}$ each to reduce the noise. The acquisition time per plant was $20 \mathrm{~min}$. A total number of 11 rosebush plants with varying architectural complexity were imaged. The output data obtained from each acquisition session is a stack of X-ray images with a pixel spacing of $0.9766 \mathrm{~mm}$ and slice spacing of $0.5 \mathrm{~mm}$. The data is represented in a 3D voxel space, where the intensity of each voxel reflected the material properties of the plant shoot at that voxel.

From the raw volume data, the 3D voxels belonging to the rosebushes and their pots were extracted through masking and thresholding. The masks were manually constructed to separate unrelated material coming from the imaging platform, and thresholding was performed to separate the plant voxels from the air. Table 1 gives the number of thresholded voxels, the number of voxels corresponding to the plant shoot, and the number of voxels on the surface of the plant shoot. The pot contains a significant portion of the voxels of the models; the large difference in the number of the voxels between models is due to different sizes of the pots. The plant shoot corresponds to the plant parts above the soil. Most of the voxels of the plant shoot are on the surface since leaves and petals and sepals of the flowers are very thin structures.

After the X-ray intensity values of the voxels corresponding to air and background material are set to zero, the remaining voxels are assigned to one of the following classes: (1) stem, (2) leaf, (3) flower, (4) pot, (5) tag. The background voxels corresponding to air were assigned "zero" values. The stem class includes both the main branches and the petioles since they have similar geometrical structures and are spatially connected. The plant shoot is composed of the stem, leaf, and flower classes.

Table 1 Number of voxels in the models (also the number of points in the corresponding point cloud)

\begin{tabular}{lcll}
\hline Model ID & $\begin{array}{l}\text { \# Thresholded } \\
\text { voxels }\end{array}$ & $\begin{array}{l}\text { \# Plant shoot } \\
\text { voxels }\end{array}$ & $\begin{array}{l}\text { \# Plant shoot } \\
\text { surface voxels }\end{array}$ \\
\hline S268650 & 794,618 & 312,212 & 275,954 \\
S268660 & 588,101 & 157,029 & 127,158 \\
S270230 & 657,195 & 205,686 & 175,800 \\
S270240 & 642,192 & 169,276 & 142,474 \\
S270250 & 818,568 & 347,013 & 301,786 \\
S271780 & $2,091,739$ & 305,534 & 264,634 \\
S271790 & $2,072,313$ & 200,346 & 171,963 \\
S271800 & $2,011,882$ & 164,108 & 138,065 \\
S273080 & $1,153,337$ & 176,155 & 145,284 \\
S273090 & $1,909,986$ & 192,755 & 166,246 \\
S273110 & $1,254,316$ & 294,528 & 257,992 \\
\hline
\end{tabular}

Figure 1 displays the thresholded X-ray volume (a), the organ-level labels obtained through annotation (b), the labels corresponding to the plant shoot (c), and the stem and petiole structure (d) of a sample rosebush model from the data set. Table 2 gives the percentages of voxels of organ classes on the plant shoot and the surface of the plant shoot.

The manual annotation was carried out with the help of ilastik (Interactive Learning and Segmentation Toolkit) [23]: Using pixel classification tool of ilastik, on a rosebush model, we manually marked several voxels in regions belonging to each class to train the classifier. Then, we obtained full-volume predictions on all models generated by the trained classifier of ilastik. Through detailed inspection, we manually corrected the labels of all voxels incorrectly labeled by ilastik.

The data set is available online at [24]. We provide the $3 \mathrm{D}$ data in the following forms: (1) the raw X-ray image stack, (2) the binary volume mask indicating the voxels of only the shoot of the plant, the tag, and the pot, and the corresponding organ-level labels, (3) the binary volume mask indicating the voxels only on the surface of the plant shoot, and the corresponding organ-level labels, (4) the point cloud composed of the points of the shoot of the plant, the tag, and the pot with colors indicating organ-level labels, (5) the point cloud composed of the points on the surface of the plant shoot with colors indicating organ-level labels. The details of the file formats and label information are explained in the Additional file 1 . Through these forms, it is possible to convert the $3 \mathrm{D}$ volumetric models to a labeled polygon mesh model and obtain 3D point clouds as viewed from any position around the plant through ray casting.

\section{Baseline methods for leaf-stem segmentation}

Vision-based plant phenotyping has been traditionally performed through analysis of $2 \mathrm{D}$ color images from which 3D characteristics of the plants (stem length, volume, leaf area, etc.) have been estimated. With the advance of 3D imaging technologies, phenotyping through the 3D capture and reconstruction of plants have gained considerable attention. In Table 3, characteristics of some of the 3D vision-based phenotyping methods that involve a segmentation stage to separate leaves from branches are summarized. 3D data was captured from various species of plants by structured light depth sensors [25, 26], laser scanners [27-31], ToF cameras [32], or from a set of color images through structure from motion $[33,34]$.

One of the disadvantages of these optics-based acquisition techniques is that they suffer from a high degree of self-occlusion of plants. As the architecture becomes more complex, more parts of the plants become heavily 


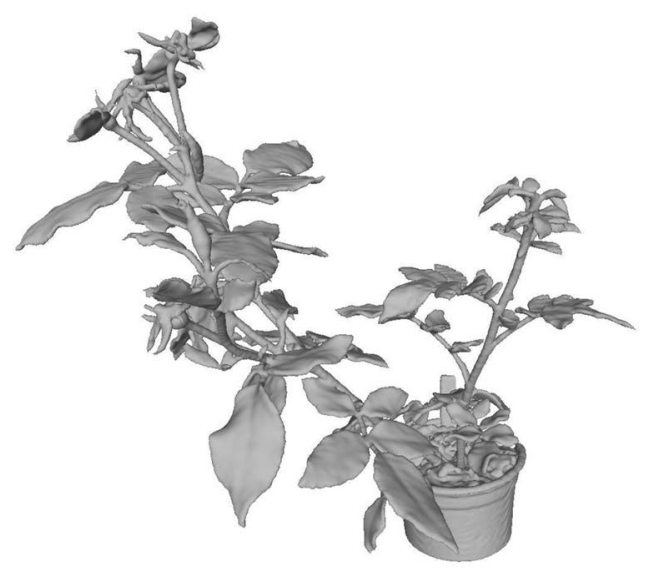

a Thresholded X-ray volume.

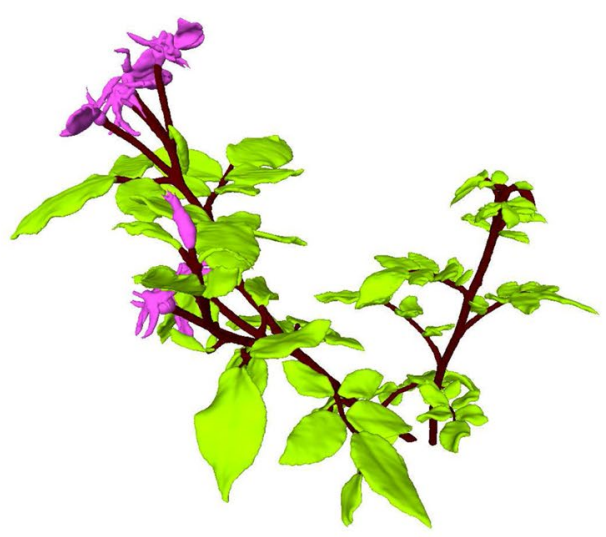

c Labels of the plant shoot.

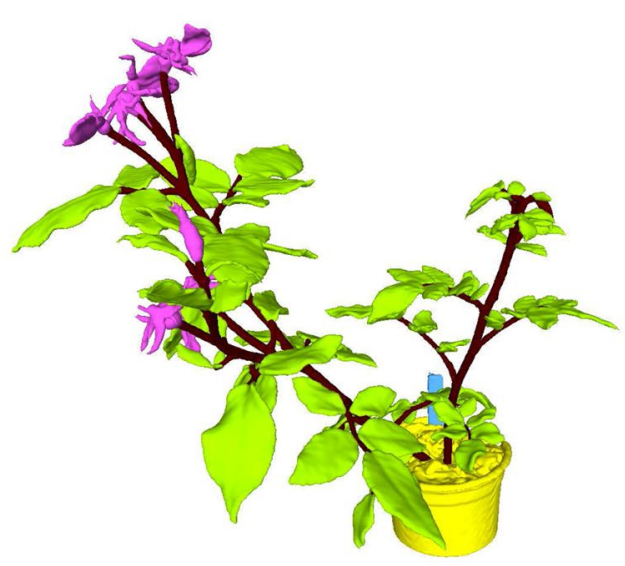

b Labels through annotation.

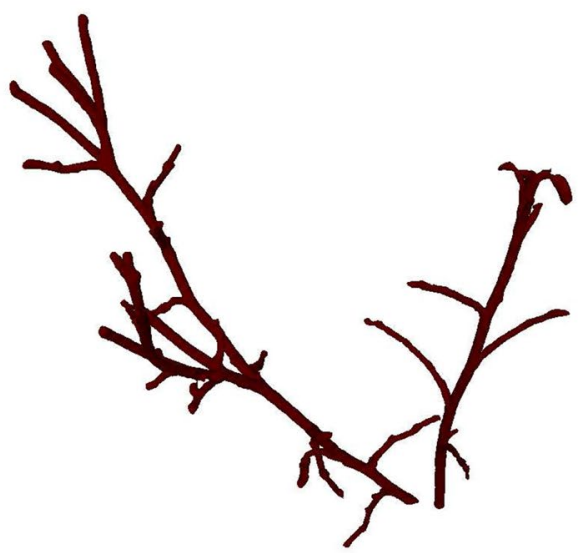

d Branches.

Fig. 1 A sample rosebush model from the data set. The raw X-ray volume is thresholded and masked to obtain the solid part shown in a. Each voxel in the volume is annotated as leaf, stem, flower, pot, or tag to obtain the ground-truth segmentation as shown in $\mathbf{b}$. In $\mathbf{c}$ only the parts corresponding to the plant shoot are shown, excluding the pot and the tag. The voxels corresponding only to stem class are shown in $\mathbf{d}$

Table 2 Percentages of voxels (points) for organ classes in the plant shoot

\begin{tabular}{|c|c|c|c|c|c|c|}
\hline Model ID & Leaf & Stem & Flower & Leaf on surface & Stem on surface & $\begin{array}{l}\text { Flower } \\
\text { on surface }\end{array}$ \\
\hline S268650 & 79.06 & 13.08 & 7.86 & 83.99 & 9.43 & 6.58 \\
\hline S268660 & 70.53 & 17.06 & 12.41 & 77.37 & 12.66 & 9.97 \\
\hline S270230 & 77.07 & 14.36 & 8.57 & 83.44 & 10.40 & 6.17 \\
\hline S270240 & 71.30 & 16.60 & 12.10 & 79.92 & 11.70 & 8.38 \\
\hline S270250 & 75.22 & 12.33 & 12.45 & 80.64 & 8.93 & 10.43 \\
\hline S271780 & 80.97 & 13.46 & 5.57 & 86.35 & 9.79 & 3.86 \\
\hline S271790 & 75.76 & 13.96 & 10.28 & 81.26 & 10.12 & 8.62 \\
\hline S271800 & 73.84 & 17.09 & 9.07 & 81.70 & 12.57 & 5.73 \\
\hline S273080 & 69.20 & 21.72 & 9.08 & 77.50 & 15.99 & 6.51 \\
\hline S273090 & 75.08 & 19.20 & 5.72 & 82.64 & 13.97 & 3.39 \\
\hline S273110 & 79.91 & 17.07 & 6.02 & 83.78 & 12.27 & 3.95 \\
\hline
\end{tabular}




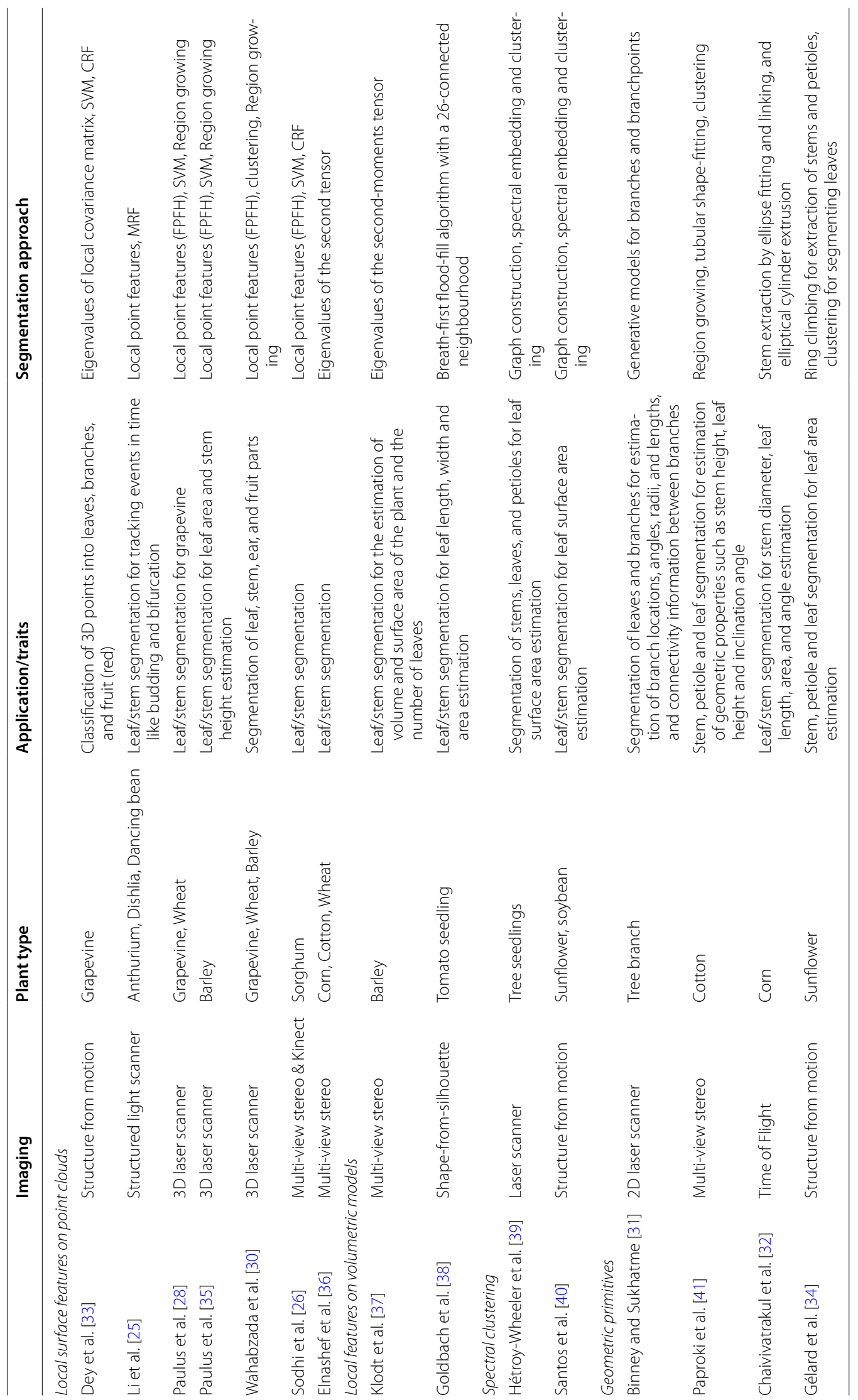


occluded, making it impossible to capture some regions from any viewpoint. That disadvantage forced most automatic part segmentation and phenotyping research to be conducted on plants with relatively simple architectural and geometrical structure, such as plants with a single stem and well-separated leaves. With X-ray imaging, 3D information of the entire plant material can be captured. However, many phenotyping activities, such as growth monitoring, require the plants not to be moved, which makes X-ray imaging impractical. The bulk of the automatic phenotyping activities is bound to rely on opticsbased acquisition devices. Although X-ray imaging will remain as an appropriate tool for applications such as root growth analysis, we envision that the ROSE-X data set will be mainly a resource for algorithms that operate on point clouds acquired with optics-based methods. The availability of complete models of real plants with high architectural complexity and full annotation will serve as a guiding resource for processing occluded point clouds of highly complicated plants acquired by RGB or depth cameras, or laser scanners.

Whether the data is in $3 \mathrm{D}$ volumetric form or is in the form of a 3D point cloud, semantic segmentation is required for particular phenotyping objectives, such as organ-level phenotyping, extraction of the architecture and event detection such as leaf growth and decay. Leafstem segmentation is the most commonly addressed problem in organ-level phenotyping. We can categorize leaf/stem segmentation methods for 3D phenotyping into the following groups: (1) segmentation using local surface features on point clouds [25, 26, 28, 30, 33, 35], (2) segmentation using local features on volumetric data [37, 38], (3) segmentation through spectral clustering [39, 40, 42], (4) segmentation by fitting geometric primitives $[31,32,34,41,43]$. Table 3 is organized using this categorization. In this work, instead of an exhaustive evaluation of all the available methods on our labeled data set, we selected four representative approaches as baseline methods for segmenting the shoot of the rosebush data into its branches and leaves. Two of these methods are based on local features extracted from the point cloud. The other two methods assume volumetric data as input, and have not been previously applied to the plant organ segmentation problem. For all methods, it is assumed that the plant shoot is already separated from the pot. In the following subsections, the baseline methods are described in detail.

\section{Segmentation using local surface features on point clouds}

One of the most common approaches to segment point clouds of plants is to use local features. Point neighborhoods on leaves and branches exhibit distinguishing distributions, which can be attributed to their sheet-like or line-like structures, respectively. One of the simplest approaches is to represent such characteristics by the eigenvalues of the covariance matrix of the neighborhood. Researchers have devised the use of more sophisticated point features such as Fast Point Feature Histograms (FPFH) $([28,35])$ that provide a rich description of the local structure around a point. In this work, we opted to use the simplest point neighborhood descriptors for the baseline methods. For more information on 3D local features, we refer to the book [44] of Laga et al.

For a point $x$ in the point cloud, the neighborhood can be defined as the set $\mathcal{N}_{\S}=\left\{x_{i}:\left\|x-x_{i}\right\|<d\right\}$, where $d$ is the radius of the neighborhood. The covariance matrix of the neighborhood is calculated as $C=\frac{1}{\left|\mathcal{N}_{\S}\right|-1} \sum_{x_{i} \in \mathcal{N}_{\S}}\left(x_{i}-\bar{x}\right)\left(x_{i}-\bar{x}\right)^{T}$, where $\bar{x}=\frac{1}{\left|\mathcal{N}_{x}\right|} \sum_{x_{i} \in \mathcal{N}_{\S}} x_{i}$ is the mean of the points.

The relative magnitudes of the eigenvalues $\left\{\lambda_{1}, \lambda_{2}, \lambda_{3}\right\}$ of the covariance matrix with $\lambda_{1} \leq \lambda_{2} \leq \lambda_{3}$ can serve as local descriptors to discriminate leaf and stem points. For a thin flat structure, we expect $\lambda_{1}$ to be much smaller than both $\lambda_{2}$ and $\lambda_{3}$. We also expect $\lambda_{2}$ and $\lambda_{3}$ to be close to each other. For a line-like structure we have a predominantly large value of $\lambda_{3}$, with $\lambda_{1}$ and $\lambda_{2}$ being much smaller.

We used the eigenvalues of the local covariance matrix in two baseline stem/leaf segmentation methods. The first is an unsupervised method based on the Markov Random Fields (MRF) formulation given in [25]. The second is a supervised method where a classifier is trained with local features derived from the eigenvalues. This second approach aligns with the methods proposed in $[26,33]$.

Local features on point clouds-unsupervised (LFPCu) : For this baseline method, we followed a simplified version of the stem/leaf classification method given in [25]. The eigenvalues are used to define local surface features on the point clouds and to search for a mapping $f_{B}$ from a point $x$ to one of the two labels for leaf $(L)$ and stem $(S)$ categories. The point cloud can be organized in a graph where the points $x \in \mathcal{X}$ correspond to the nodes and pairs of locally connected points $\left(x_{i}, x_{j}\right) \in \mathcal{E}$ constitute the edges. In our implementation, a pair $\left(x_{i}, x_{j}\right)$ was considered to be an edge if the Euclidean distance between them is less than $1.4 \mathrm{~mm}$. The energy associated with a particular label mapping is defined as

$$
E\left(f_{B}\right)=w_{D} \sum_{x \in \mathcal{X}} D_{x}\left(f_{B}(x)\right)+w_{V} \sum_{\left(x_{i}, x_{j}\right) \in \mathcal{E}} V\left(f_{B}\left(x_{i}\right), f_{B}\left(x_{j}\right)\right) .
$$

The weight factors $w_{D}$ and $w_{V}$ determine a compromise between the class likelihoods of individual nodes and the coherence across the edges. $D_{x}\left(f_{B}(x)\right)$ corresponds to the data term (the unary potential) which gives the cost of 
classifying a point $x$ into a leaf or stem category. The term $V\left(f_{B}\left(x_{i}\right), f_{B}\left(x_{j}\right)\right)$ gives the smoothness term (the pairwise potential) and is used to encourage labeling coherence between neighboring points. The energy function is minimized through min-cut algorithm [45] to obtain the optimum labels for the point cloud.

To determine the data and smoothness terms, an estimate of the curvature at point $x$ is computed using the eigenvalues of the covariance matrix as $C(x)=\frac{\lambda_{1}}{\lambda_{1}+\lambda_{2}+\lambda_{2}}$. The range of the curvature values is $[0,1 / 3]$, and leaf points are expected to have lower curvature values than stem points. A flatness feature is defined as $R(x)=\log \left(\max (C(x)), c_{\epsilon}\right)$, where $c_{\epsilon}$ is set to $0.015 . R(x)$ is in the range $\left[R_{L}, R_{S}\right]$ with $R_{L}=\log \left(c_{\epsilon}\right)$ and $R_{S}=\log (1 / 3)$. Then, the data term is calculated as

$$
D_{x}\left(f_{B}(x)\right)=\left\{\begin{array}{l}
R(x)-R_{L}, \text { if } f_{B}(x)=L . \\
R_{S}-R(x), \text { if } f_{B}(x)=S .
\end{array}\right.
$$

The smoothness term also depends on the curvature $C(x)$, which is used as a measure of the discontinuity of the surface. The pairwise potential is set to be inversely proportional to the curvature since a high curvature value indicates a discontinuity which can be considered as the boundary of a plant part. The smoothness term is defined as

$$
V\left(f_{B}\left(x_{i}\right), f_{B}\left(x_{j}\right)\right)= \begin{cases}\max \left(\frac{1}{C\left(x_{i}\right)}, \frac{1}{C\left(x_{j}\right)}\right), & \text { if } f_{B}\left(x_{i}\right) \neq f_{B}\left(x_{j}\right) . \\ 0, & \text { if } f_{B}\left(x_{i}\right)=f_{B}\left(x_{j}\right) .\end{cases}
$$

Notice that this method is an unsupervised method in the sense that it does not require labeled training data to transform or organize features to boost their discriminating power. However, the weight factors $w_{D}$ and $w_{V}$ in Eq. (1) need to be fixed. Through experimentation on one rosebush reserved to train the methods, we found that $w_{D}=0.9$ and $w_{N}=0.1$ yielded the best results.

Local features on point clouds-supervised (LFPC-s): For the second baseline method, we selected to derive local features from the eigenvalues of the local covariance matrix, and used SVM as the classifier as in the work of Dey et al. ([33]). We defined the local features as follows:

$$
F_{1}=\frac{\lambda_{1}}{\sqrt{\lambda_{2} \lambda_{3}}} \quad F_{2}=\frac{\lambda_{2}}{\lambda_{3}} \quad F_{3}=\frac{\lambda_{1}}{\sqrt{\lambda_{1} \lambda_{2} \lambda_{3}}} \quad F_{4}=\frac{\lambda_{1}}{\lambda_{2}}
$$

The size of the neighborhood from which the eigenvalues are computed determines the scale at which the local structures will be analyzed. The stem and the petioles of the plant shoot have varying widths, likewise the leaves exhibit a large size variability. Instead of fixing the radius, we extracted the features $\left\{F_{1}, F_{2}, F_{3}, F_{4}\right\}$ at various scales and concatenate them into a single feature vector. In our tests, we used six scales, corresponding to neighborhoods of radii $2,3,4,5,6$, and $7 \mathrm{~mm}$. Using one of the rosebush models with ground truth labels, we trained a two-class linear SVM classifier.

\section{Segmentation using local features on volumetric data (LFVD)}

The point cloud data acquired from optic-based sensors such as RGB cameras or laser scanners can be converted to binary volumetric data using a 3D occupancy grid. The regular structure of 3D volume allows to apply standard filtering and feature extraction tools such as smoothing and estimation of first and second order derivatives. The software ilastik [23] can extract various types of features from 3D volume data: the color features correspond to the raw intensity values smoothed by a Gaussian filter. The edge features are the eigenvalues of the structure tensor, eigenvalues of the Hessian matrix, the gradient magnitude of the difference of Gaussians and Laplacian of Gaussian. The texture features correspond to eigenvalues of the structure tensor, eigenvalues of the Hessian matrix, and orientation features are the raw structure tensor and Hessian matrix entries. In our tests, the mentioned features are extracted from data smoothed by Gaussian filters with scales $0.7,1.0,1.6,3.5,5.0$, and $10.0 \mathrm{~mm}$.

The voxels of the original X-ray data possess intensity values which are determined by the intensity of the $\mathrm{X}$-rays passing through the voxels and the material properties. X-ray intensity values in our models depend on the material properties of plant parts; e.g., leaves have very low intensity values compared to branches. In order to have comparable results between the volume-based and surface-based baseline methods, we used the binary volume mask, indicating the voxels of only the shoot of the plant. We further set the values of the voxels which are not on the surface of the plant-shoot, i.e., interior voxels, to zero, so that only the voxels on the surface of the plantshoot will remain.

We employed ilastik [23] to extract intensity, edge, and texture features from one binary plant model and to train a random forest classifier [46] using the ground-truth labels. Once the classifier is trained on one model; it is tested on all the other models on the data set.

\section{CNN on volume data (3DU-Net)}

As a representative of deep learning methods, we selected 3D U-Net [47], which is originally proposed to provide dense volumetric segmentation maps for biomedical images. It is an extension of the 2D U-net architecture developed by Ronneberger et al. [48]; all the 2D operations in the 2D u-net are replaced with their 3D counterparts. The input volume is first passed through an analysis path with four resolution layers, each of which is composed of two $3 \times 3 \times 3$ convolutions with 
Rectified Linear Units (reLU) and one $3 \times 3 \times 3 \max$ pooling operation. Max pooling corresponds to downsampling by using the maximum value from each of a cluster of neurons at the prior layer. Then a synthesis path is applied with four resolution layers each consisting of one $2 \times 2 \times 2$ upconvolution operator followed by two $2 \times 2 \times 2$ convolutions with reLU. The high-resolution features obtained at the analysis path are also provided to the synthesis path through shortcut connections between layers of equal resolution. The size of the input voxel grid to the network is $144 \times 144 \times 144$, and the output is a volumetric data of the same size giving the label of each voxel. The architecture graph can be found in [47]. For more information on deep learning and the definitions of the classical layers that constitute the basis of deep neural networks, we refer to the book [49] of Goodfellow et al.

As we did with the baseline method based on local volumetric features, we only used the thresholded voxels on the surface of the shoot, so the input is binary devoid of the intensity information. We used one rosebush model to train the network. We extracted 25 subvolumes of size $144 \times 144 \times 144$ from various locations of the full volume of the model such that each subvolume contained leaf and stem instances. 20 of the subvolumes were used for training and 5 of them were used for validation. For a test model, we regularly partitioned the volume to non-overlapping subvolumes and provided the subvolumes to the network as inputs to get the corresponding segmentation.

\section{Results}

In this paper, we concentrated on the problem of partitioning the plant models into its leaf and stem (branch) parts; so the training and evaluation of the baseline methods are performed using the ground truth labels corresponding to the leaves and stems only. In our evaluation, we ignored the predictions generated on the flower parts.

There are many metrics for segmentation evaluation, such as Matthews Correlation Coefficient [50], Cohen's $\kappa$ coefficient [51], Dice Similarity Coefficient [52], all with their advantages and all applicable in the framework of our benchmark. In this paper, we used precision (also known as Positive Predictive Value), recall (also known as sensitivity), and Intersection over Union
(IoU) to evaluate the baseline methods. Recall for the leaf class $\left(R_{\text {leaf }}\right)$ is the ratio of the number of correctly labeled leaves (true positives) to the total number of leaf points in the ground truth (true positives + false negatives). Precision for the leaf class $\left(P_{\text {leaf }}\right)$ is the ratio of the number of correctly labeled leaves (true positives) to the total number of points classified as leaf points by the algorithm (true positives + false positives). Recall $\left(R_{\text {stem }}\right)$ and precision $\left(P_{\text {stem }}\right)$ for the stem class are defined in the same way. Intersection over Union (IoU) metric for each class $\left(\mathrm{IoU}_{\text {leaf }}\right.$ and $\left.\mathrm{IoU}_{\text {stem }}\right)$ is defined as the ratio of all the true positives to the sum of true positives, false negatives and false positives.

For a single fold of the experimental evaluation, we selected one rosebush model for training and tested the algorithms on the remaining 10 models. For the unsupervised method based on local features on point clouds, we used the training model to optimize the weights of the data and smoothness terms. The results were averaged over the test models and over 5 -fold experiments. A different rosebush model is reserved as training data for each fold. Table 4 gives the performances of the baseline leaf/stem segmentation methods. The visual results for a sample test rosebush are given in Fig. 2. The predicted labels of the rosebush model are displayed as a volume or as a point cloud depending on the type of the data the corresponding method processes. Figure 3 gives the stem points predicted by each baseline method. Correct predictions of the stem points with their connectivity maintained are especially important for establishing the architectural structure of the plant.

We can observe from Table 4 and Fig. 2 that the voxel classification method through local features (LFVD) gives the best overall performance for leaf/stem classification. It is a supervised method combining multi-scale volumetric local features with the random forest classifier. For this particular data set, it can model well the scale variations of leaf and stem points as well as their geometrical variations due to their locations on the organ (in the middle or at the border). The recall rate for the stem class is around $90 \%$, meaning that $10 \%$ of the points on the branches are missed. Most missed stem points are on the petioles, which are in between close leaflets and possess an almost planar structure (Fig. 4c). The

Table 4 Performances of the baseline leaf/stem segmentation methods (\%)

\begin{tabular}{llllll}
\hline Method & $\boldsymbol{R}_{\text {leaf }}$ & $\boldsymbol{R}_{\text {stem }}$ & $\boldsymbol{P}_{\text {leaf }}$ & $\boldsymbol{P}_{\text {stem }}$ & IoU $_{\text {stem }}$ \\
\hline LFPC-u & $95.74 \pm 1.74$ & $88.03 \pm 1.82$ & $98.23 \pm 0.33$ & $75.01 \pm 9.76$ & $94.10 \pm 1.54$ \\
LFPC-S & $97.79 \pm 0.46$ & $80.50 \pm 1.29$ & $97.19 \pm 0.48$ & $83.67 \pm 4.88$ & $95.10 \pm 0.46$ \\
LFVD & $99.38 \pm 0.13$ & $90.01 \pm 1.17$ & $98.53 \pm 0.43$ & $95.38 \pm 1.02$ & $97.93 \pm 0.47$ \\
3D U-Net & $81.06 \pm 1.68$ & $97.41 \pm 1.43$ & $99.63 \pm 0.29$ & $54.0 \pm 5.77$ & $86.23 \pm 0.31$ \\
\hline
\end{tabular}




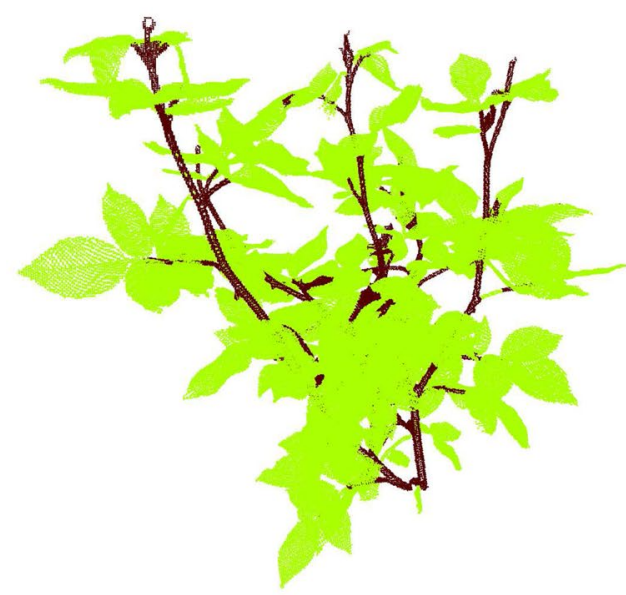

a Segmentation result with LFPC-u

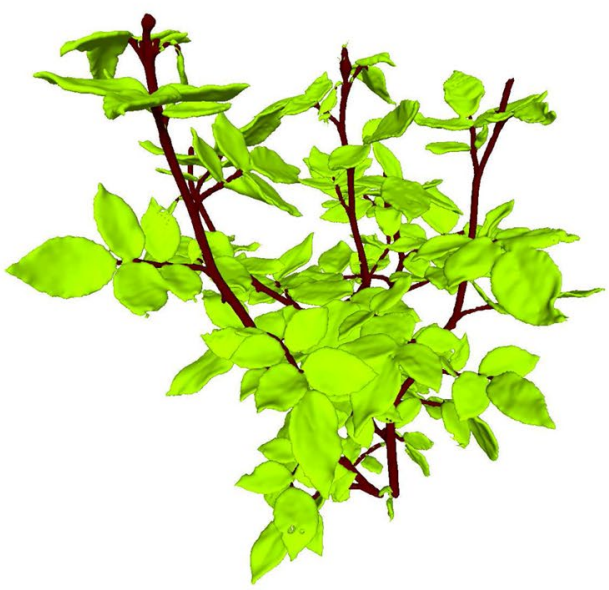

c Segmentation result with LFVD

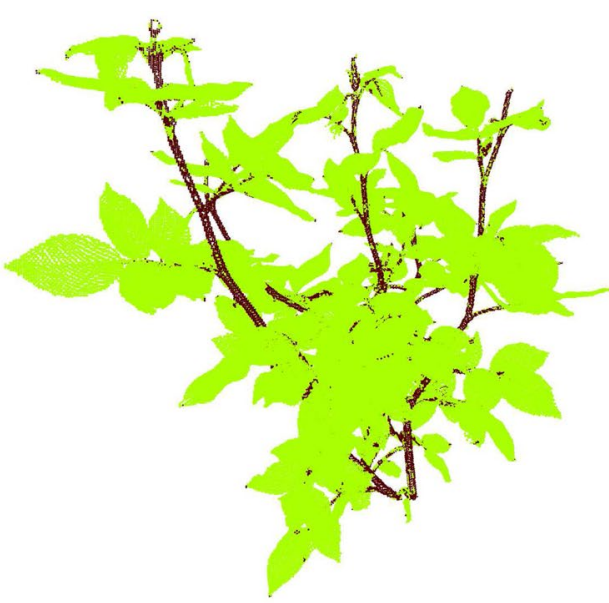

b Segmentation result with LFPC-s

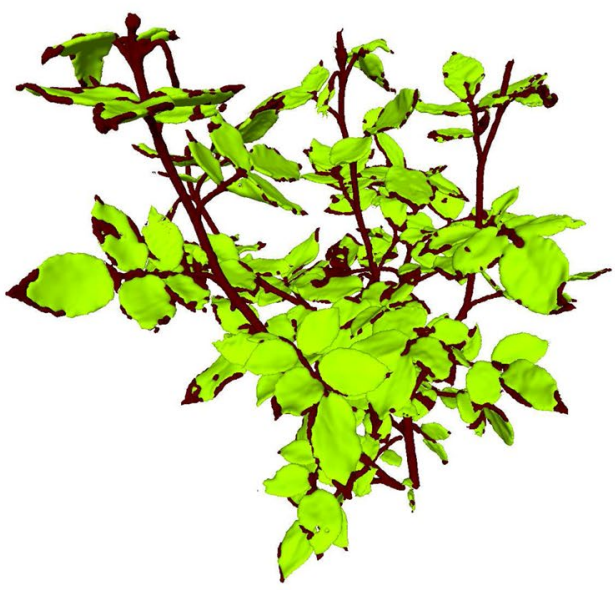

d Segmentation result with 3D U-Net

Fig. 2 Leaf and stem labels predicted by the baseline methods for a sample test rosebush. The rendering is in volumetric form for LFVD and 3D U-Net and in point cloud for LFPC-u and LFPC-s. The methods LFPC-u (a) and LFVD (c) produced smooth results, while the labels predicted by LFPC-s (b) are slightly noisy. 3D U-Net (d) wrongly classifies leaf borders as stems

discontinuities in the stem-branch structure predicted by LFVD (Fig. 3c) generally correspond to the petiole portions just in between opposite leaflets.

The classification results obtained by LFPC- $\mathrm{u}$ are smooth (Fig. 2a) and the stem structure is mostly connected (Fig. 3a) due to the regularization imposed by the MRF formulation. However, smoothing labels of adjacent points in regions of low curvature leads to an entire leaf or a portion of it to be classified as stem if there is a smooth transition of normals at the boundary as seen in Fig. 4a. This propagation of labels through boundaries with low curvature causes a relatively low stem precision rate (Table 4). Likewise, smooth petiole and leaf boundaries lead to the classification of petiole points as leaves affecting the stem recall rate negatively. Although this method is unsupervised in the sense that it does not involve a classifier that learns feature transformations through labeled training data, the weights of the data and smoothness terms in Eq. 1 should be optimized for different plant species.

The performance of LFPC-s is slightly higher than that of LFPC-u in terms of the IoU metric (Table 4). Notice that we did not incorporate the MRF formulation for the baseline method LFPC-s, although it is completely applicable through setting the data term using SVM scores. Since no smoothness constraint is imposed on the labels, we can observe isolated noisy predictions along the stem and on the leaves (Fig. 2b). The predicted stem structure 


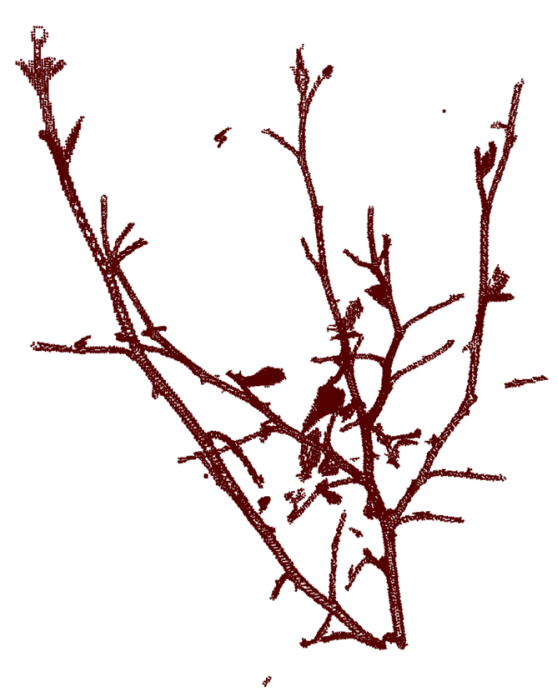

a Stems predicted by LFPC-u

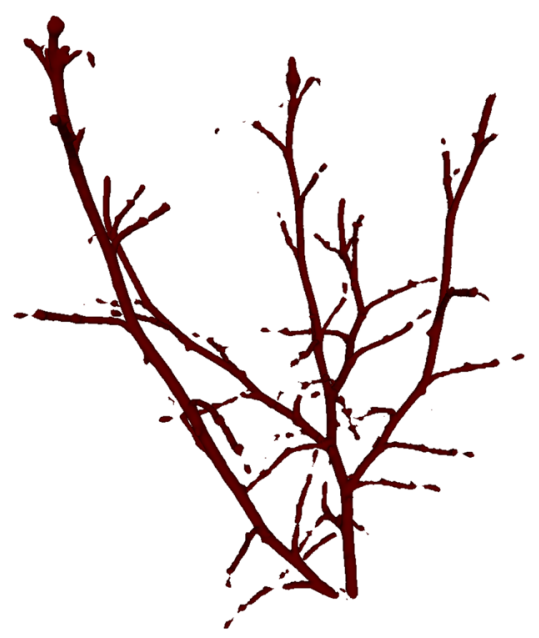

c Stems predicted by LFVD

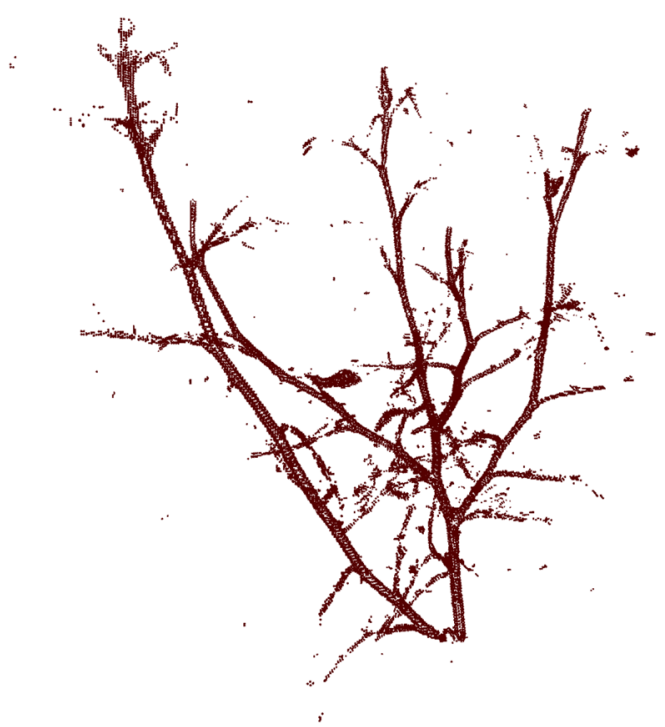

b Stems predicted by LFPC-s

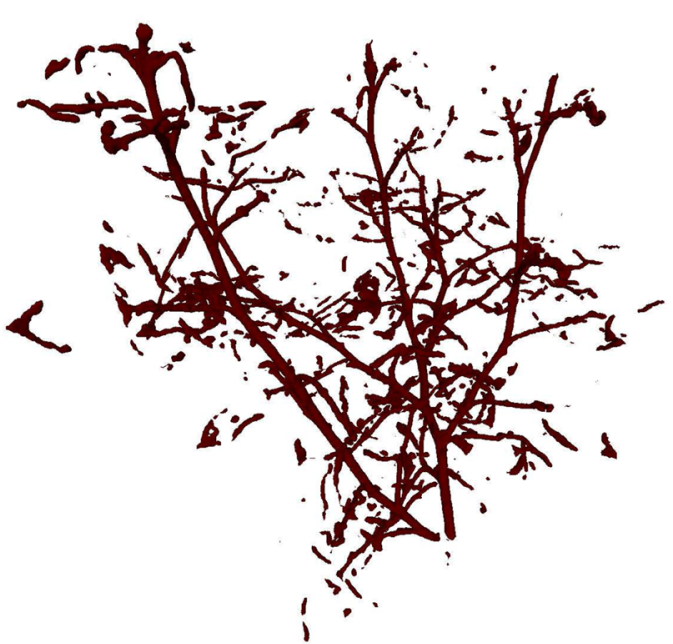

d Stems predicted by 3D U-Net

Fig. 3 Stem labels predicted by the baseline methods for a sample test rosebush. The rendering is in volumetric form for LFVD and 3D U-Net and in point cloud for LFPC-u and LFPC-s. With the methods LFPC-u (a) and LFVD (c) the predicted stem structure is mostly connected, while LFVD (c) misses some petiole portions. The noisy predictions produced by the method LFPC-s (b) are more visible here. 3D U-Net (d) classifies large portions of leaves as stems

has unconnected small regions due to some leaf points classified as stems (Fig. 4b). Most of these errors occur at the midribs which are usually the thickest parts of the leaves.

3D U-net gives the lowest performance as compared to the other methods. Boundaries and thick portions of leaves are classified as stems as can be observed from Fig. 4d. We give in Fig. 5 the evolution of the training and validation loss. The dice coefficient function is used as the loss function in 3D U-Net algorithm, which shows a value in a range of 0 to 1 . In this case, a negative is multiplied to values for optimization purposes. The curves in this figure show that the model can converge fast after about 50 epochs with the minimum overfitting between training and validation. However, the CNN network did not model the variations of leaves since we used sub-volumes 


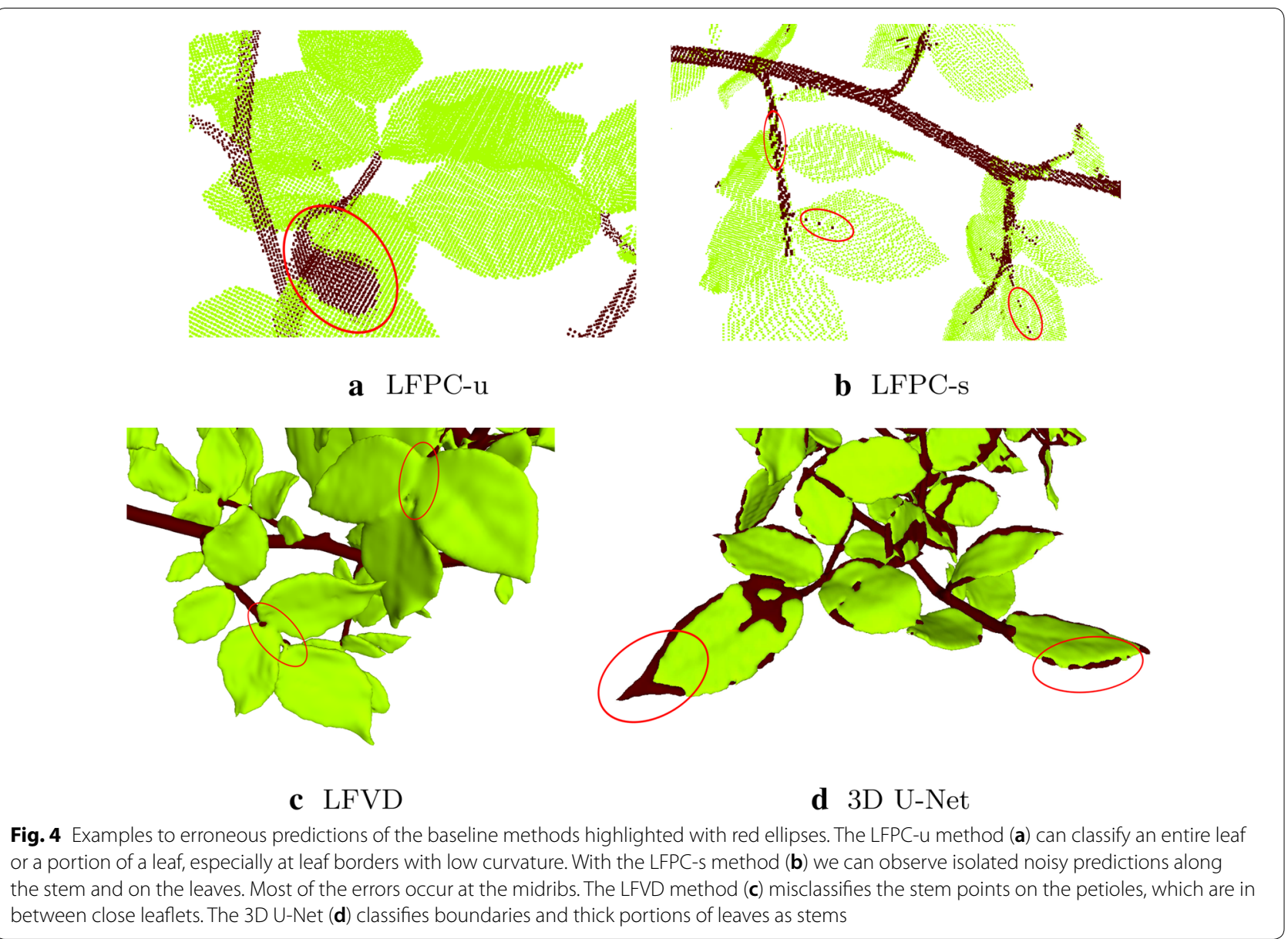

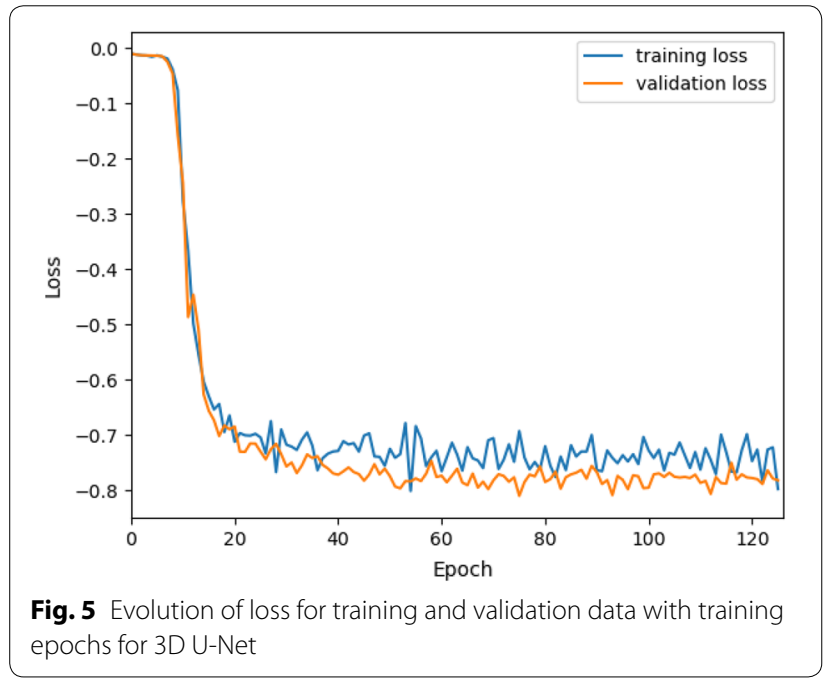

from a single rosebush model for training to have a fair comparison with other baseline supervised methods. The $3 \mathrm{D} U$-net has far more parameters to learn than the other methods; therefore, more training data is required for it to be properly trained. Besides, we directly applied the original 3D U-net architecture [47], which was designed for bio-medical data, without modification. In order to improve the results with deep learning, one can either increase the training data by using more than one rosebush model, employ data augmentation strategies, alter the 3D U-Net architecture or propose a new architecture suitable for 3D segmentation of plants. However, detailed analysis of the modifications on these lines is beyond the main objective of this work. We leave the design of 3D $\mathrm{CNN}$ architectures specific to plant organ segmentation as an open research problem, to the solution of which our entire labeled data set can contribute.

The methods LFPC-u, LFPC-s, and LFVD were run on a computer with an Intel processor of $3.5 \mathrm{GHz}$ and 128 GB RAM. LFPC-u and LFPC-s were coded with MATLAB, while LFVD was implemented with Python. The average processing time for segmentation of a single model with LFPC-u is $4.2 \mathrm{~min}$. The training time of the SVM classifier for LFPC-s is $5.1 \mathrm{~min}$ on average. The segmentation time for a test model with LFPC-s is $1.6 \mathrm{~min}$. 
The training time of the Random Forest classifier for LFVD is $13.4 \mathrm{~min}$, and the testing time is $3.3 \mathrm{~min}$. The 3D U-Net was trained using Python on a computer with an Intel processor of $2.2 \mathrm{GHz}$ and $8 \mathrm{GPUs}$ of $64 \mathrm{~GB}$. The training time is $3 \mathrm{~h}$, while segmentation time for a new test model is $4.3 \mathrm{~min}$ on average.

\section{Discussion}

The ROSE-X data set includes high resolution 3D models of real rosebush plants, each of which was annotated at the voxel level with the corresponding botanical organ class. In this article, we focused on the step of segmentation of leaves and stems of automatic phenotyping pipelines. We provided a benchmark for proper comparison of current and future approaches for leaf/stem segmentation.

In this article, the focus has been on leaf segmentation from the stem. This is the first essential step in analyzing the shape and the architecture of the plant. Other questions can be addressed with the ROSE-X data set including issues raised by breeders, producers or consumers such as the study of interactions between genotype and environment on the one hand and phenotype and visual perception on the other. Such issues require the analysis of the growth and morphogenesis of the plant through effective phenotyping. With this objective in mind, it is possible to consider petiole segmentation, the distinction between leaflet and leaves, the detection of meristem along the stem, the analysis of the different part of the flower and the stage of development.

Also, the extraction and encoding of the architectural structure of the plant in the form of an organized collection of the main stem, second and higher order branches, and the branching locations is an important phenotyping task. Another task would be to extract geometrical characteristics of the individual architectural components and their spatial relationships, such as the length and width of the branch segments, petioles and their branching angles, leaf length, width, and area, together with the leaf inclination angles. These advanced botanical traits would be accessible with the spatial resolution of the 3D images of the proposed data set ROSE-X.

In order to evaluate the accuracy of phenotyping methods that aim to extract such more advanced botanical traits, we will release a forthcoming extension of the data set, with extended ground truth data in the form of geometrical properties of individual organs such as leaves, leaflets, petioles, stem segments, branching locations, and the spatial relationship between them.

We present the rosebush models in volumetric form, however, our main concern is to provide labeled data of plants with complex architecture for phenotyping methods that use the visible surface points of the plants as input. The conversion of the volumetric form to a point cloud via sampling or via ray casting from an arbitrary viewpoint is straightforward. As part of the future work, we will generate partial point clouds from the models as seen from around the plant, and apply phenotyping methods that rely on partial data.

Another important issue is the applicability of leaf/ stem classification methods trained with the rosebush data set to other plant species. Future work will involve the expansion of the data set with 3D models of different species, and the adaptation of the classifiers learned from one species to others.

\section{Conclusion}

This paper introduces a data set composed of $11 \mathrm{com}$ plete 3D models acquired through X-ray scanning of real rosebush plants. The models are stored in a voxel grid structure. We also provide the ground truth data, where each voxel stores the corresponding organ class label. The plant models are free from self-occlusion, however they posses complex architectural structure. As a sample application where the data set can be of use, we chose leaf-stem segmentation and compared the classification performances of four baseline methods. We observed that the volumetric approach (LFVD), where a random forest classifier is trained with local features, yielded the best performance. However, other baseline methods tested in this work are also open to further improvement, and there are yet the state-of-the-art techniques (Table 3) to be evaluated on our dataset. The data set is suitable to be annotated with more advanced traits and can be used as a benchmark for evaluation of automatic phenotyping methods that go beyond classifying plant points as leaves and stems.

\section{Supplementary information}

Supplementary information accompanies this paper at https://doi. org/10.1186/s13007-020-00573-w.

Additional file 1. Description of the provided annotated dataset.

Acknowledgements

Auhtors thank Paul Salat for contribution in fine curation of the data set.

Authors' contributions

HD and DR conceived and designed this study. GG and DR carried out the acquisition of $X$-ray images. HD performed the implementation and analyzed the result of the image segmentation methods. PR contributed in the process of the data via the 3D U-Net. HD and DR wrote and revised the manuscript. All authors read and approved the final manuscript.

Funding

Helin Dutagaci and Pejman Rasti gratefully acknowledge Région des Pays de la Loire and Angers Loire Métropole for the funding of their Post-Doc positions. 


\section{Availability of data and materials}

Entire data set will be released after acceptance on a website of University of Angers. Only a single sample is made available in the submitted manuscript.

\section{Competing interests}

The authors declare that they have no competing interests.

\section{Ethics approval and consent to participate}

Not applicable for that section.

\section{Consent for publication}

Not applicable for that section.

\begin{abstract}
Author details
1 LARIS, UMR INRA IRHS, Université d'Angers, 62 Avenue Notre Dame du Lac, 49000 Angers, France. ${ }^{2}$ INRA, UMR1345 Institut de Recherche en Horticulture et Semences, 42 Georges Morel CS 60057, 49071 Beaucouze, France. ${ }^{3}$ ESAIP, école d'ingénieur informatique et environnement, Saint Barthélemy d'Anjou, France.
\end{abstract}

Received: 2 August 2019 Accepted: 21 February 2020

Published online: 04 March 2020

\section{References}

1. Goodfellow I, Bengio Y, Courville A. Deep learning. Cambridge: MIT Press; 2016.

2. Deng J, Dong W, Socher R, Li L, Li K, Fei-Fei L. Imagenet: a large-scale hierarchical image database. In: 2009 IEEE conference on computer vision and pattern recognition; 2009, p. 248-55. https://doi.org/10.1109/ CVPR.2009.5206848.

3. Lobet $G$, Draye $X$, Périlleux C. An online database for plant image analysis software tools. Plant Methods. 2013;9(1):38. https://doi. org/10.1186/1746-4811-9-38.

4. Chitwood DH, Otoni WC. Morphometric analysis of Passiflora leaves: the relationship between landmarks of the vasculature and elliptical Fourier descriptors of the blade. GigaScience. 2017; https://doi.org/10.1093/ gigascience/giw008.

5. Minervini M, Fischbach A, Scharr H, Tsaftaris SA. Finely-grained annotated datasets for image-based plant phenotyping. Pattern Recognit Lett. 2016;81:80-9. https://doi.org/10.1016/j.patrec.2015.10.013.

6. Veley KM, Berry JC, Fentress SJ, Schachtman DP, Baxter I, Bart R. Highthroughput profiling and analysis of plant responses over time to abiotic stress. bioRxiv. 2017; . https://doi.org/10.1101/132787.

7. Uchiyama H, Sakurai S, Mishima M, Arita D, Okayasu T, Shimada A, Taniguchi R. An easy-to-setup 3D phenotyping platform for KOMATSUNA dataset. In: 2017 IEEE international conference on computer vision workshops (ICCVW); 2017, p. 2038-45. https://doi.org/10.1109/ICCVW.2017.239.

8. Cruz JA, Yin X, Liu X, Imran SM, Morris DD, Kramer DM, Chen J. Multimodality imagery database for plant phenotyping. Mach Vis Appl. 2016;27(5):735-49. https://doi.org/10.1007/s00138-015-0734-6.

9. Bernotas G, Scorza LCT, Hansen MF, Hales IJ, Halliday KJ, Smith LN, Smith ML, McCormick AJ. A photometric stereo-based 3D imaging system using computer vision and deep learning for tracking plant growth GigaScience 2019;8(5). https://doi.org/10.1093/gigascience/giz056.giz05 6. http://oup.prod.sis.lan/gigascience/article-pdf/8/5/giz056/28704193/ giz056.pdf.

10. Wen W, Guo X, Wang Y, Zhao C, Liao W. Constructing a three-dimensional resource database of plants using measured in situ morphological data. Appl Eng Agric. 2017;33(6):747-56. https://doi.org/10.13031/aea.12135.

11. Perez-Sanz F, Navarro PJ, Egea-Cortines M. Plant phenomics: an overview of image acquisition technologies and image data analysis algorithms. GigaScience. 2017; . https://doi.org/10.1093/gigascience/gix092.

12. Atkinson JA, Pound MP, Bennett MJ, Wells DM. Uncovering the hidden half of plants using new advances in root phenotyping. Curr Opin Biotechnol. 2019;55:1-8. https://doi.org/10.1016/j.copbio.2018.06.002.

13. Staedler YM, Masson D, Schönenberger J. Plant tissues in 3D via x-ray tomography: simple contrasting methods allow high resolution imaging. PLOS ONE. 2013;8(9):1-10. https://doi.org/10.1371/journal.pone.0075295.
14. Hughes N, Askew K, Scotson CP, Williams K, Sauze C, Corke F, Doonan $J$, Nibau C. Non-destructive, high-content analysis of wheat grain traits using X-ray micro computed tomography. Plant Methods. 2017;13(1):76. https://doi.org/10.1186/s13007-017-0229-8.

15. Gomez FE, Carvalho G, Shi F, Muliana AH, Rooney WL. High throughput phenotyping of morpho-anatomical stem properties using X-ray computed tomography in sorghum. Plant Methods. 2018;14(1):59. https://doi. org/10.1186/s13007-018-0326-3.

16. Du J, Zhang Y, Guo X, Ma L, Shao M, Pan X, Zhao C. Micron-scale phenotyping quantification and three-dimensional microstructure reconstruction of vascular bundles within maize stalks based on micro-CT scanning. Funct Plant Biol. 2017;44(1):10-22. https://doi.org/10.1071/FP16117.

17. Schneider JV, Rabenstein R, Wesenberg J, Wesche K, Zizka G, Habersetzer J. Improved non-destructive 2D and 3D X-ray imaging of leaf venation. Plant Methods. 2018;14(1):7. https://doi.org/10.1186/s13007-018-0274-y.

18. Wang Z, Verboven P, Nicolai B. Contrast-enhanced 3D micro-CT of plant tissues using different impregnation techniques. Plant Methods. 2017;13(1):105. https://doi.org/10.1186/s13007-017-0256-5.

19. Mathers AW, Hepworth C, Baillie AL, Sloan J, Jones H, Lundgren M, Fleming AJ, Mooney SJ, Sturrock CJ. Investigating the microstructure of plant leaves in 3D with lab-based X-ray computed tomography. Plant Methods. 2018;14(1):99. https://doi.org/10.1186/s13007-018-0367-7.

20. Garbez M, Chéné Y, Belin É, Sigogne M, Labatte J-M, Hunault G, Symoneaux R, Rousseau D, Galopin G. Predicting sensorial attribute scores of ornamental plants assessed in $3 \mathrm{D}$ through rotation on video by image analysis: a study on the morphology of virtual rose bushes. Comput Electron Agric. 2016;121:331-46. https://doi.org/10.1016/j.compa g.2016.01.001.

21. Chéné Y, Rousseau D, Belin É, Garbez M, Galopin G, Chapeau-Blondeau F. Shape descriptors to characterize the shoot of entire plant from multiple side views of a motorized depth sensor. Mach Vis Appl. 2016;27(4):44761. https://doi.org/10.1007/s00138-016-0762-x.

22. Vázquez-Arellano M, Griepentrog H, Reiser D, Paraforos D. 3-D imaging systems for agricultural applications—a review. Sensors. 2016; . https:// doi.org/10.3390/s16050618.

23. Sommer C, Strähle C, Köthe U, Hamprecht FA. llastik: Interactive learning and segmentation toolkit. In: Eighth IEEE international symposium on biomedical imaging (ISBI 2011). Proceedings; 2011, p. 230-3. https://doi. org/10.1109/ISBI.2011.5872394.

24. The ROSE-X Dataset. https://uabox.univ-angers.fr/index.php/s/rnPm5 EHFK6Xym9t.

25. Li Y, Fan X, Mitra NJ, Chamovitz D, Cohen-Or D, Chen B. Analyzing growing plants from 4D point cloud data. ACM Trans Graph. 2013;32(6):157. https://doi.org/10.1145/2508363.2508368.

26. Sodhi P, Vijayarangan S, Wettergreen D. In-field segmentation and identification of plant structures using 3D imaging. In: 2017 IEEE/RSJ international conference on intelligent robots and systems (IROS); 2017, p. 5180-7. https://doi.org/10.1109/IROS.2017.8206407.

27. Paulus S, Behmann J, Mahlein A-K, Plümer L, Kuhlmann H. Low-cost 3D systems: suitable tools for plant phenotyping. Sensors. 2014;14(2):300118. https://doi.org/10.3390/s140203001.

28. Paulus S, Dupuis J, Mahlein A-K, Kuhlmann H. Surface feature based classification of plant organs from 3D laserscanned point clouds for plant phenotyping. BMC Bioinformatics. 2013;14(1):238. https://doi. org/10.1186/1471-2105-14-238.

29. Chaudhury A, Brophy M, Barron JL. Junction-based correspondence estimation of plant point cloud data using subgraph matching. IEEE Geosci Remote Sens Lett. 2016;13(8):1119-23. https://doi.org/10.1109/ LGRS.2016.2571121.

30. Wahabzada M, Paulus S, Kersting K, Mahlein A-K. Automated interpretation of 3D laserscanned point clouds for plant organ segmentation. BMC Bioinformatics. 2015;16(1):248. https://doi.org/10.1186/s1285 9-015-0665-2.

31. Binney J, Sukhatme GS. 3D tree reconstruction from laser range data. In: 2009 IEEE international conference on robotics and automation; 2009, p. 1321-6. https://doi.org/10.1109/ROBOT.2009.5152684.

32. Chaivivatrakul S, Tang L, Dailey MN, Nakarmi AD. Automatic morphological trait characterization for corn plants via 3D holographic reconstruction. Comput Electron Agric. 2014;109:109-23. https://doi.org/10.1016/j. compag.2014.09.005. 
33. Dey D, Mummert L, Sukthankar R. Classification of plant structures from uncalibrated image sequences. In: 2012 IEEE workshop on the applications of computer vision (WACV); 2012, p. 329-36. https://doi. org/10.1109/WACV.2012.6163017.

34. Gélard W, Devy M, Herbulot A, Burger, P. Model-based segmentation of 3D point clouds for phenotyping sunflower plants. In: Proceedings of the 12th international joint conference on computer vision, imaging and computer graphics theory and applications — volume 4: VISAPP, (VISIGRAPP 2017); 2017, p. 459-67. https://doi.org/10.5220/0006126404 590467.

35. Paulus S, Dupuis J, Riedel S, Kuhlmann H. Automated analysis of barley organs using 3D laser scanning: an approach for high throughput phenotyping. Sensors. 2014;14(7):12670-86. https://doi.org/10.3390/s1407 12670.

36. Elnashef B, Filin S, Lati RN. Tensor-based classification and segmentation of three-dimensional point clouds for organ-level plant phenotyping and growth analysis. Comput Electron Agric. 2019;156:51-61. https://doi. org/10.1016/j.compag.2018.10.036.

37. Klodt $M$, Cremers D. High-resolution plant shape measurements from multi-view stereo reconstruction. In: Agapito L, Bronstein MM, Rother C, editors. Computer vision-ECCV 2014 workshops. Springer, Cham; 2015, p. 174-84

38. Golbach F, Kootstra G, Damjanovic S, Otten G, van de Zedde R. Validation of plant part measurements using a $3 \mathrm{~d}$ reconstruction method suitable for high-throughput seedling phenotyping. Mach Vis Appl. 2016;27(5):663-80. https://doi.org/10.1007/s00138-015-0727-5.

39. Hétroy-Wheeler F, Casella E, Boltcheva D. Segmentation of tree seedling point clouds into elementary units. Int J Remote Sens. 2016;37(13):2881907. https://doi.org/10.1080/01431161.2016.1190988.

40. Santos TT, Koenigkan LV, Barbedo JGA, Rodrigues GC. 3D plant modeling: localization, mapping and segmentation for plant phenotyping using a single hand-held camera. In: Agapito L, Bronstein MM, Rother C, editors. Computer vision-ECCV 2014 workshops. Springer, Cham; 2015, p. 247-63.

41. Paproki A, Sirault X, Berry S, Furbank R, Fripp J. A novel mesh processing based technique for 3D plant analysis. BMC Plant Biol. 2012;12(1):63. https ://doi.org/10.1186/1471-2229-12-63.

42. Boltcheva D, Casella E, Cumont R, Hétroy F. A spectral clustering approach of vegetation components for describing plant topology and geometry from terrestrial waveform LiDAR data. In: Lintunen A, editor. 7th international conference on functional-structural plant models, Saariselkä, Finland; 2013. https://doi.org/10.13140/2.1.1114.1928.

43. Nguyen CV, Fripp J, Lovell DR, Furbank R, Kuffner P, Daily H, Sirault X. 3D scanning system for automatic high-resolution plant phenotyping. In: 2016 international conference on digital image computing: techniques and applications (DICTA); 2016, p. 1-8. https://doi.org/10.1109/DICTA .2016.7796984.

44. Laga H, Guo Y, Tabia H, Fisher R, Bennamoun M. 3D Shape analysis: fundamentals, theory, and applications. Hoboken: Wiley-Blackwell; 2019.

45. Boykov Y, Kolmogorov V. An experimental comparison of min-cut/maxflow algorithms for energy minimization in vision. IEEE Trans Pattern Anal Mach Intell. 2004;26(9):1124-37. https://doi.org/10.1109/TPAMI.2004.60.

46. Breiman L. Random forests. Mach Learn. 2001;45(1):5-32. https://doi. org/10.1023/A:1010933404324.

47. Çiçek Ö, Abdulkadir A, Lienkamp SS, Brox T, Ronneberger O. 3D U-net: learning dense volumetric segmentation from sparse annotation. In: Ourselin S, Joskowicz L, Sabuncu MR, Unal G, Wells W, editors. Medical image computing and computer-assisted intervention-MICCAI 2016. Cham: Springer; 2016. p. 424-32.

48. Ronneberger O, Fischer P, Brox T. U-Net: convolutional networks for biomedical image segmentation. In: Navab N, Hornegger J, Wells WM, Frangi AF, editors. Medical image computing and computer-assisted intervention-MICCAI 2015. Cham: Springer; 2015. p. 234-41.

49. Goodfellow I, Bengio Y, Courville A. Deep learning. Cambridge: The MIT Press; 2016.

50. Powers DMW. Evaluation: from precision, recall and F-measure to ROC, informedness, markedness \& correlation. J Mach Learn Technol. 2011;2(1):37-63.

51. Cohen J. Weighted kappa: nominal scale agreement provision for scaled disagreement or partial credit. Psychol Bull. 1968;70(4):213-20. https:// doi.org/10.1037/h0026256.

52. Dice LR. Measures of the amount of ecologic association between species. Ecology. 1945;26(3):297-302. https://doi.org/10.2307/1932409.

\section{Publisher's Note}

Springer Nature remains neutral with regard to jurisdictional claims in published maps and institutional affiliations.
Ready to submit your research? Choose BMC and benefit from:

- fast, convenient online submission

- thorough peer review by experienced researchers in your field

- rapid publication on acceptance

- support for research data, including large and complex data types

- gold Open Access which fosters wider collaboration and increased citations

- maximum visibility for your research: over 100M website views per year

At BMC, research is always in progress.

Learn more biomedcentral.com/submissions 